\title{
Belgeo
}

Revue belge de géographie

$4 \mid 2019$

Les politiques de mobilité urbaine en Europe après la crise : adaptations et innovations

\section{Les mutations des politiques de déplacement dans les villes moyennes : des ambitions aux contraintes de l'action. L'exemple de Périgueux}

Une analyse à partir du projet de pôle d'échanges

Mobility policies mutations in mid-sized cities: from ambitions to action. The example of Périgueux. An analysis based on the interchange station project

\section{Emilie Roudier}

\section{(2) OpenEdition}

\section{Journals}

Édition électronique

URL : http://journals.openedition.org/belgeo/36696

DOI : $10.4000 /$ belgeo.36696

ISSN : 2294-9135

Éditeur :

National Committee of Geography of Belgium, Société Royale Belge de Géographie

Référence électronique

Emilie Roudier, «Les mutations des politiques de déplacement dans les villes moyennes : des ambitions aux contraintes de l'action. L'exemple de Périqueux », Belgeo [En ligne], 4 | 2019, mis en ligne le 12 février 2020, consulté le 20 juillet 2020. URL : http://journals.openedition.org/belgeo/36696 ; DOI : https://doi.org/10.4000/belgeo.36696

Ce document a été généré automatiquement le 20 juillet 2020.

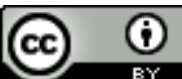

Belgeo est mis à disposition selon les termes de la licence Creative Commons Attribution 4.0 International. 


\section{Les mutations des politiques de déplacement dans les villes moyennes : des ambitions aux contraintes de l'action. L'exemple de Périgueux}

Une analyse à partir du projet de pôle d'échanges

Mobility policies mutations in mid-sized cities: from ambitions to action. The example of Périgueux. An analysis based on the interchange station project

Emilie Roudier

\section{Introduction}

La crise des villes moyennes est au cœur des débats politiques et académiques. En parallèle du plan "Action cœur de ville ", lancé par le gouvernement en décembre 2017, de nombreux travaux récents analysent les mutations et les difficultés de ces territoires, situés à un échelon intermédiaire entre les métropoles régionales et les petits bourgs urbains (Santamaria, 2012). Tous soulignent peu ou prou la diversité des crises auxquelles ces villes doivent faire face (géopolitique, économique, territoriale, etc.) tout en dressant un état des lieux nuancé des situations locales (Béhar, 2018; Delpirou, 2019).

2 Cet article s'intéresse plus particulièrement à la crise des transports des villes moyennes. Ces territoires se caractérisent non seulement par une part modale élevée de la voiture, utilisée en moyenne pour deux tiers des déplacements (Gart, 2015), mais également par une forte dépendance à l'automobile (Dupuy, 1999). L'absence de congestion routière et de contrainte de stationnement facilite, d'une part, l'usage de la voiture et la périurbanisation croissante de leurs agglomérations complexifie, d'autre 
part, le développement de réseaux de transports collectifs urbains depuis plus de trente ans (Charmes, 2017). Cette situation de monopole de la voiture tend toutefois à accroître leur vulnérabilité énergétique et à dégrader, de plus en plus, leur cadre de vie (Lejoux, 2017).

3 Face à l'augmentation des nuisances locales, les politiques des villes moyennes évoluent progressivement depuis les années 2000 et accordent une place plus grande à la planification des déplacements $\left(\right.$ Cerema, 2019) ${ }^{2}$. Les multiples injonctions nationales à la mobilité durable, portées notamment par la loi Solidarité et Renouvellement Urbain ${ }^{3}$ et la loi Grenelle ${ }^{4}$, ainsi que le renforcement des compétences intercommunales en matière de transport, ont contribué à cette évolution (Gallez, 2007). Après être longtemps restées peu coercitives vis-à-vis de l'automobile (Héran, 2017), les intercommunalités de ces territoires mettent de plus en plus en œuvre des actions pour réduire l'usage de la voiture et pour développer l'usage des modes alternatifs à la voiture individuelle, moins émetteurs d'émissions de gaz à effet de serre (transports collectifs, vélo, marche à pied, etc.).

4 Dans ce contexte, nombre d'entre elles choisissent de transformer leurs gares ferroviaires pour aménager des pôles d'échanges multimodaux (PEM) : depuis dix ans, plus de soixante projets de PEM ont ainsi été lancés et/ou réalisés dans les villes moyennes (figure 1). Ces projets visent à réaménager les espaces situés aux abords de la gare (parvis, parkings vélos, parkings voiture, gare routière, etc.) dans le but de favoriser les correspondances entre les modes de transport et l'usage du train, en facilitant l'accès à la gare ferroviaire (Menerault, 2006; Richer, 2008). Ils sont généralement conduits par les intercommunalités en partenariat avec les différents échelons des collectivités territoriales (état, région, commune) et les acteurs ferroviaires (SNCF Gares \& Connexions, SNCF Réseau), compte tenu de la fragmentation des compétences transport et aménagement et des propriétés foncières autour des gares (Maulat, Passalaqua, 2014). 
Figure 1. Les projets de pôles d'échanges dans les villes moyennes (2009-2019).

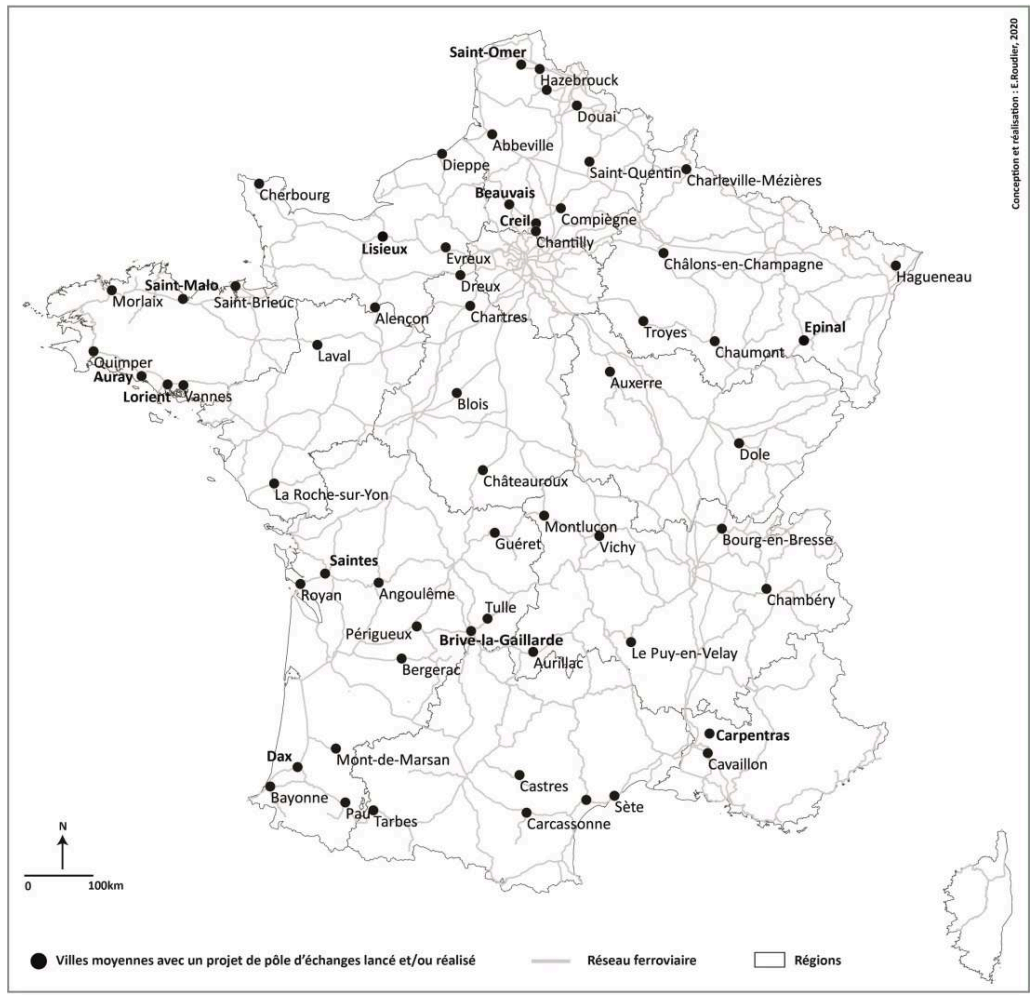

Emilie Roudier, 2020

5 Cet article propose de mettre en perspective la multiplication de ces projets avec les évolutions récentes des politiques locales de déplacement. Il analyse plus précisément la conduite des projets de pôle d'échanges dans les villes moyennes, les objectifs politiques qui y sont associés et leurs places dans la planification locale : à quelles problématiques locales répondent ces projets ? Leur conduite est-elle associée à des changements de référentiels dans les politiques locales? Marque-t-elle une mise à l'agenda des mobilités dans les villes moyennes depuis dix ans? Les intercommunalités ont-elles pour autant les capacités d'action pour mener à bien leurs projets de pôles d'échanges et leurs politiques locales?

6 Le parti pris de cet article est de considérer les projets de pôle d'échanges multimodaux comme des projets d'aménagement et comme des prismes d'analyse des politiques locales. A partir de cette approche, ces projets sont considérés comme des outils destinés à mettre en œuvre et transcrire spatialement des orientations politiques (Pinson, 2005) et comme des supports pour étudier les évolutions de ces orientations. Ils constituent des entrées intéressantes pour étudier la conduite de la politique locale de mobilité car ils sont souvent initiés par les acteurs locaux, ils portent sur tous les modes de transport et ils sont généralement associés à des enjeux complexes, notamment techniques (exploitation ferroviaire, multiplicité des objectifs, etc.) et financiers (coûts élevés, convention multipartenariale, etc.).

Dans les villes moyennes, les intercommunalités disposent en effet de ressources modestes en ingénierie et financement face à la baisse constante des dotations territoriales depuis 2007 et à l'augmentation du poids des charges de centralité (De Roo, 2005 ; Santamaria, 2012). 
8 L'analyse se concentre sur une étude de cas: le cas de Périgueux, ville moyenne du département de la Dordogne, en région Nouvelle Aquitaine (figure 2). Elle s'intéresse à la politique locale de mobilité et au projet de pôle d'échanges en gare de Périgueux lancé en 2014 par la Communauté d'Agglomération du Grand Périgueux. Cette étude de cas ne saurait certes représenter l'ensemble des villes moyennes françaises, qui se caractérisent par une diversité de contextes locaux et par des dynamiques territoriales contrastées (Demazière, 2014). De même, les configurations et la desserte des gares varient d'une ville moyenne à l'autre, avec des conséquences sur la fréquentation des trains et les objectifs de ces projets. Ceci étant, cette étude de cas détaillée pourra permettre, d'une part, d'appréhender une partie des enjeux des mobilités dans les villes moyennes et, d'autre part, d'analyser précisément l'évolution de la politique locale de déplacement en parallèle de la conduite du projet de pôle d'échanges en gare de Périgueux.

Figure 2. La situation territoriale de la commune de Périgueux.

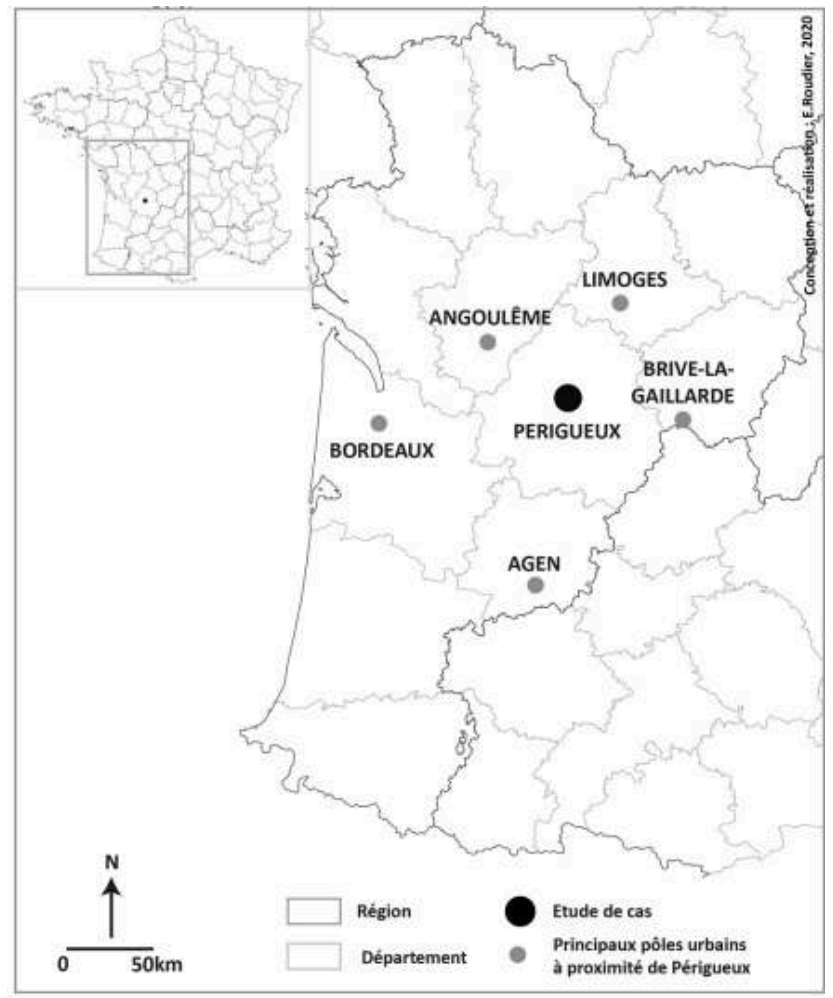

Emilie Roudier, 2020

9 L'étude se fonde sur une enquête réalisée en immersion dans un bureau d'études qui a participé aux études amont du projet de pôle d'échanges entre 2014 et $2015^{5}$. Elle s'appuie sur l'analyse de documents institutionnels locaux (plan global de déplacement, plan local d'urbanisme) et de documents techniques issus de l'étude (cahier des charges, entretiens, rapports, comptes-rendus). Elle a été complétée par une dizaine d'entretiens semi-directifs réalisés avec la majorité des parties prenantes du projet (commune, intercommunalité, région, SNCF Gares \& Connexions) et des observations de terrain entre 2015 et 2018. 


\section{L'agglomération périgourdine face au règne de la voiture}

10 La politique locale de déplacement est définie et mise en œuvre par l'intercommunalité, la Communauté d'Agglomération du Grand Périgueux. Afin de comprendre les objectifs de cette politique intercommunale, il convient de revenir sur la situation locale de Périgueux et de réaliser une analyse fine - mais non exhaustive - des pratiques de déplacements et des mutations territoriales qui y sont associées. L'analyse se concentre principalement sur la commune de Périgueux et les communes limitrophes qui constituent le cœur urbain de l'agglomération, avec plus de deux tiers des habitants de l'intercommunalité. La forte périurbanisation de l'agglomération et la pression accrue de la voiture dans les déplacements quotidiens sont notamment deux des principales problématiques sur lesquelles se fondent cette politique locale.

\section{Une ville-centre en crise et en concurrence avec ses périphéries}

11 A mi-chemin entre Bordeaux et Limoges, Périgueux est le principal pôle urbain et la préfecture du département de la Dordogne (29912 habitants) ${ }^{6}$. A l'instar de nombreuses villes moyennes, ce statut politique lui confère des fonctions traditionnelles de commandement et d'animation locale (Béhar, 2011). Il a aussi contribué à l'aménagement de voies de communication majeures au cours du XIX ${ }^{\mathrm{e}}$ siècle qui font de Périgueux un nœud de transport important du département (Genty, 1981).

Périgueux est un carrefour routier et ferroviaire (figure 3). La commune se situe historiquement sur la route reliant Bordeaux à Limoges qui longe la vallée de l'Isle. Dès le début du XIXe, sa desserte est ensuite complétée avec l'aménagement de plusieurs traversées vers Angoulême, Brive et Bergerac, et plus récemment avec l'A89 reliant Bordeaux à Lyon (Tesson, 2002). C'est dans la deuxième partie du XIX ${ }^{e}$ siècle que la commune devient aussi un carrefour ferroviaire avec la construction de plusieurs axes permettant de relier Bordeaux, Limoges, Brive-la-Gaillarde et Agen. Elle bénéficie d'une des meilleures dessertes ferroviaires du département, avec des dessertes nord-sud et ouest-est, même si elle n'a pas réussi à obtenir de liaison directe vers Paris, abandonnée au profit de Brive-la-Gaillarde (Genty, 1981). A cette époque, un port est aussi aménagé à Périgueux sur les bords de l'Isle, qui est la principale voie de navigation entre le département de la Dordogne et l'Atlantique. 
Figure 3. Périgueux : un carrefour routier et ferroviaire.

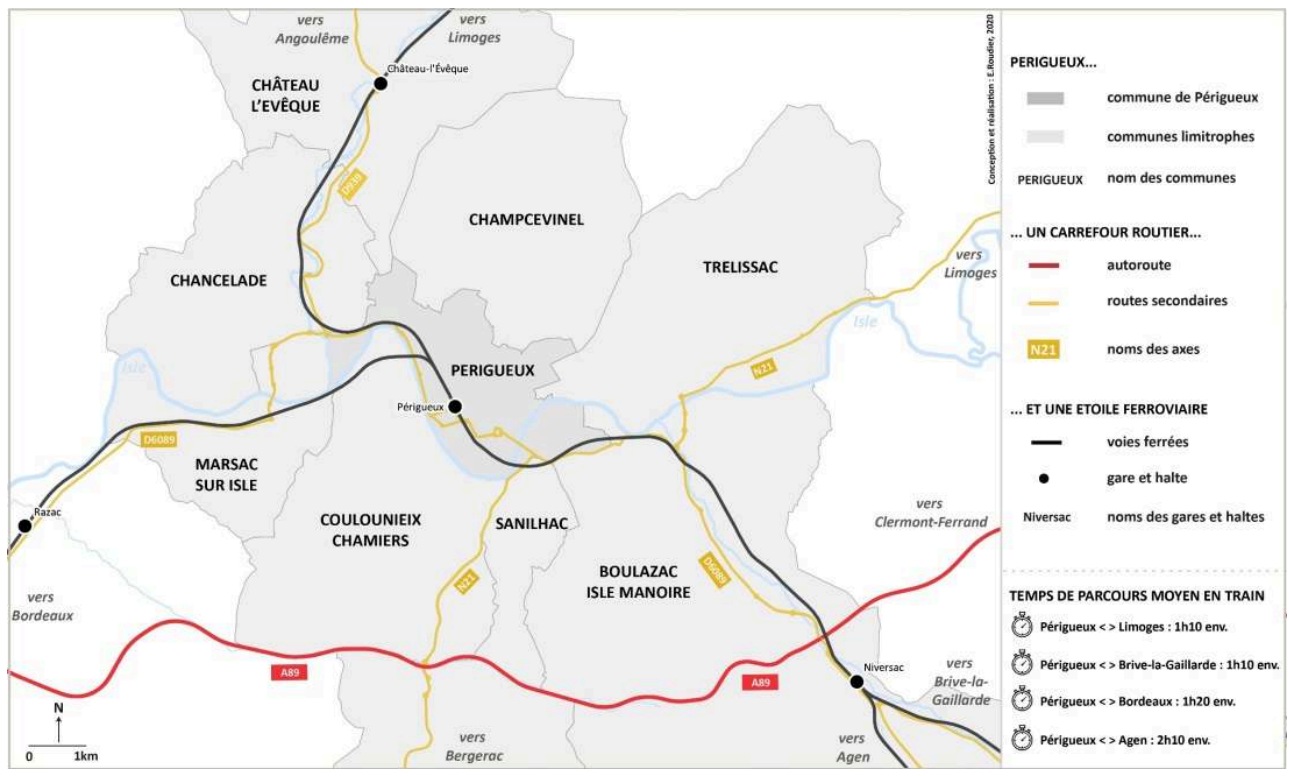

Emilie Roudier, 2019

13 Le maillage dense des réseaux de transports a largement contribué au rayonnement économique de la commune. Dès la deuxième partie du XIX $\mathrm{X}^{\mathrm{e}}$ siècle, le port permet l'acheminement de nombreuses marchandises et des industries, spécialisées dans la métallurgie et la construction, s'installent à Périgueux (Chadefaud, 1963). La mise en service des ateliers SNCF, dédiés principalement à la réparation et la maintenance des trains, en 1864 marque par ailleurs un tournant dans l'économie locale. Employant jusqu'à 25000 ouvriers, les ateliers ont contribué à la croissance des activités économiques de Périgueux et à la croissance de la population communale avec l'arrivée de nouveaux ouvriers (Eloi, 2005).

Ces fonctions historiques sont toutefois en crise. Depuis les années 1960, à l'instar de la plupart des villes moyennes françaises, l'attractivité de Périgueux est déstabilisée par le double mouvement de métropolisation et de périurbanisation, qui tend à concentrer les hommes et les activités dans les métropoles et dans les communes périurbaines (De Roo, 2005). Les populations des communes situées aux portes de Périgueux augmentent depuis les années 1960 (Di Méo, 1971) tandis que la population de la commune de Périgueux baisse fortement entre 1960 et le début des années 2010 (figure 4). De nouveaux pôles de commerces et d'emplois sont même aménagés dans ces communes de première couronne, le long des principaux axes routiers (Hays, 1993). En parallèle, le phénomène de désindustrialisation observé à l'échelle nationale a particulièrement touché Périgueux. La baisse du nombre d'emplois a entraîné une baisse du nombre d'habitants, des fermetures d'établissements et une augmentation des établissements en friches. Face à ces mutations territoriales, la commune de Périgueux reste toutefois une centralité commerciale locale, avec un faible taux de vacance dans son centre-ville - autour de $5 \%$ entre 2001 et 2014 - et une centralité économique en étant le principal pôle d'emploi de l'agglomération et du département ${ }^{7}$. 
Figure 4. Périgueux : une ville-centre concurrencée par l'attractivité résidentielle de ses périphéries.

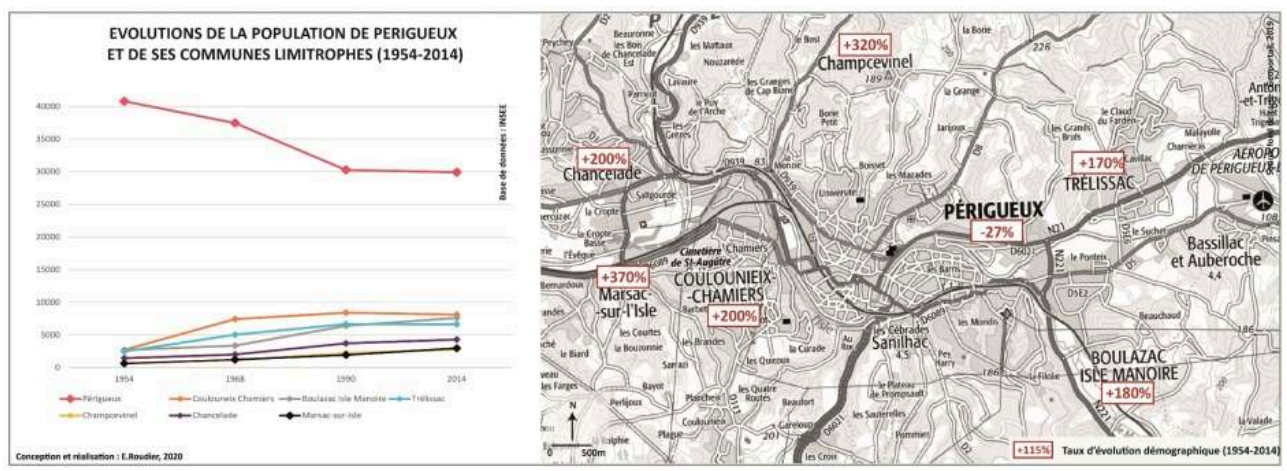

Emilie Roudier, 2020

\section{Une agglomération dépendante de la voiture}

Ces mutations territoriales sont en partie liées aux pratiques locales de déplacement. La démocratisation de l'automobile à partir des années 1960 a notamment facilité les localisations résidentielles dans les communes périurbaines, tout comme le développement des infrastructures routières qui a aussi contribué à réduire les rapports distance-temps (Dupuy, 1999).

Dans l'agglomération périgourdine, la part de la voiture dans les déplacements est largement dominante par rapport aux autres modes : elle est utilisée pour réaliser plus de deux tiers des déplacements $(72 \%)^{8}$. Cette part modale correspond peu ou prou à celle observée dans les villes de taille similaire et reste beaucoup plus forte que dans les grandes villes où la part modale de la voiture oscille entre $50 \%$ et $60 \%$ et a même tendance à baisser ces dernières années (Gart, 2015). Le maillage dense du réseau routier permet une bonne accessibilité aux communes périurbaines et des liaisons rapides vers Périgueux qui concentre les principaux pôles générateurs de déplacements (pôles d'études, d'emplois et de services). Près de deux tiers des déplacements domiciletravail dans l'agglomération se concentrent vers Périgueux et vers ses communes limitrophes (figure 5). Cette polarisation des flux vers Périgueux, couplée à l'intensification constante de la circulation automobile dans l'agglomération, tend néanmoins à augmenter les nuisances liées au trafic automobile, en particulier dans le centre de Périgueux (forte congestion en heures de pointe, pollution, encombrement de la voirie, etc.) $)^{9}$. 
Figure 5. Périgueux : le premier pôle d'études et d'emplois de l'agglomération.

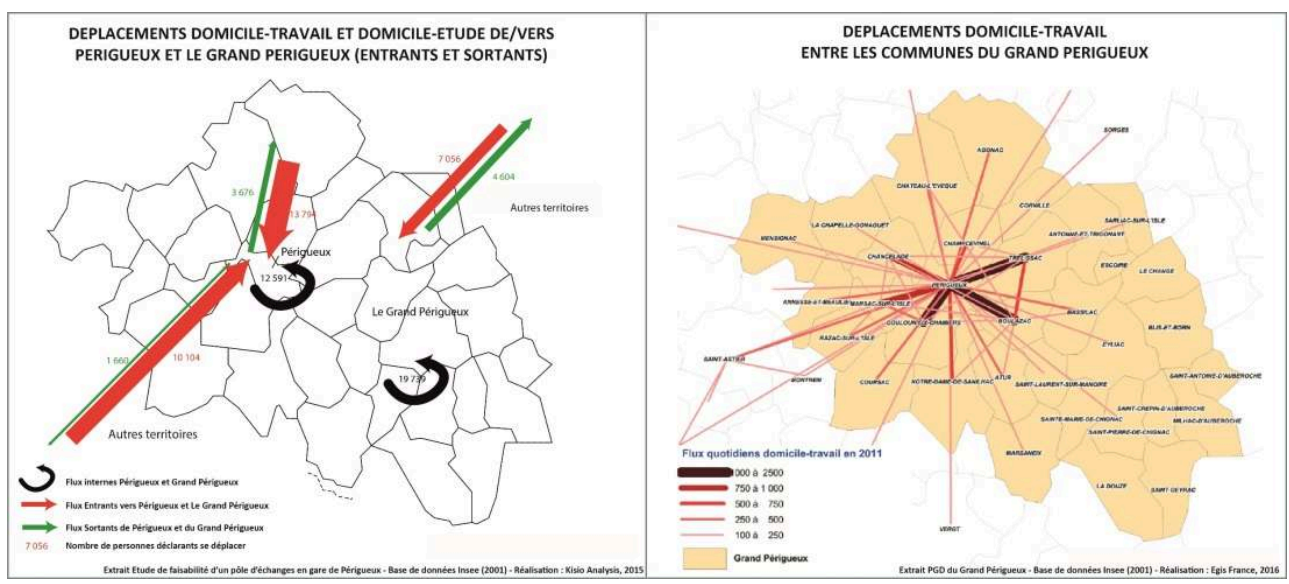

Emilie Roudier, 2020

17 A une échelle plus fine, cette part modale de la voiture témoigne de la dépendance à la mobilité routière de l'agglomération face à la faiblesse des offres et des aménagements dédiés aux modes alternatifs (Lejoux, 2017). Le réseau de bus urbains de l'agglomération périgourdine, composé d'une vingtaine de lignes avant 2014, est peu utilisé au quotidien ( $3 \%$ des déplacements domicile-travail). D'une part, le manque de performance de l'offre par rapport à la voiture décourage son utilisation (temps de parcours, itinéraires, faible desserte en soirée et le week-end, etc.). D'autre part, l'étalement urbain dans les communes périurbaines, éloignées de Périgueux, complexifie aussi la configuration des lignes et des dessertes dans ces communes. A l'instar des transports collectifs, la pratique du vélo et de la marche à pied est aussi relativement faible, notamment pour les trajets domicile-travail (respectivement $4 \%$ et $9 \%$ ). Dans les deux cas, l'absence d'aménagements cyclables et piétons dédiés sur les principaux axes routiers et dans le centre de Périgueux est considérée comme un frein à ces mobilités ${ }^{10}$.

L'agglomération périgourdine bénéficie toutefois d'un maillage ferroviaire relativement dense. Avec plus de 850000 voyageurs annuels et environ 3000 voyageurs au quotidien, la gare de Périgueux est la plus fréquentée de l'agglomération et du département. La gare est principalement fréquentée par des actifs et par des lycéens et étudiants : plus de $80 \%$ des voyageurs se rendent sur leur lieu d'études ou d'emplois. Elle n'occupe pas pour autant une position centrale dans la commune de Périgueux : le site de la gare est à un kilomètre du centre-ville, dans la partie ouest du tissu urbain (figure 6) ${ }^{11}$. Sa desserte est assurée au quotidien par une soixantaine de trains express régionaux et trains d'équilibre du territoire. L'agglomération compte également sept autres gares et haltes aménagées sur les quatre axes ferroviaires qui traversent l'agglomération, dont les principales se situent à Niversac, Razac et Château l'Evêque ${ }^{12}$. 
Figure 6. Une gare en périphérie immédiate du centre-ville de Périgueux.

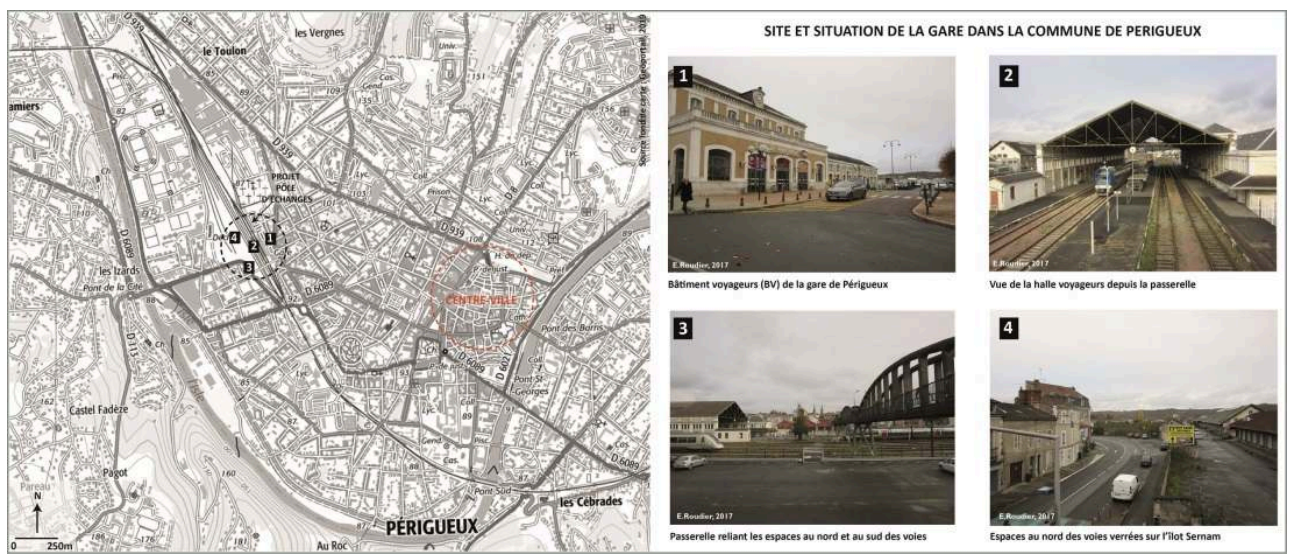

Emilie Roudier, 2020

Cette analyse montre que Périgueux est une centralité locale et départementale, qui s'est historiquement développée et organisée autour de l'usage de la voiture. Le constat d'une dégradation des conditions de vie et de déplacement dans la commune et son cœur urbain incite toutefois progressivement les acteurs locaux à mettre en œuvre une planification des mobilités sur le territoire, de façon à maîtriser l'usage de l'automobile et à en réduire les nuisances. Des premières mesures sont mises en œuvre par l'intercommunalité, notamment à partir de 2008 avec la réalisation d'un plan de déplacement urbain volontaire et la restructuration de l'offre urbaine. Ce n'est cependant qu'en 2014 que la question de la mobilité est pleinement mise à l'agenda de la politique intercommunale.

\section{Le projet de pôle d'échanges : un tournant dans la politique de mobilité périgourdine}

L'année 2014 marque un changement dans l'organisation de l'intercommunalité : la Communauté d'Agglomération Périgourdine devient la Communauté d'Agglomération du Grand Périgueux et a un périmètre d'action élargi ${ }^{13}$. La lutte contre la circulation automobile dans le centre de Périgueux devient une priorité de sa politique de déplacement et, plus largement, de son mandat. Ce faisant, la nouvelle communauté d'agglomération met en place des actions pour faire évoluer la circulation et les pratiques de déplacement, en conduisant notamment le projet d'aménagement d'un pôle d'échanges aux abords de la gare de Périgueux dès avril 2014. L'analyse des conditions de lancement de ce projet montre qu'il cristallise à plusieurs niveaux les recompositions des ambitions et des objectifs politiques de l'intercommunalité vis-à-vis des déplacements.

\section{Le levier pour favoriser le report modal vers les transports collectifs}

Le lancement de ce projet s'inscrit à l'origine dans une stratégie de recomposition de l'offre en transports collectifs, incluant l'offre urbaine et l'offre ferroviaire. Cette double évolution a pour objectif d'améliorer la qualité de l'offre dans l'agglomération et pour ambition d'augmenter leurs fréquentations. 

l'intercommunalité pour compléter l'offre de bus urbain existante. Cette solution est souvent privilégiée par les villes moyennes pour améliorer la vitesse commerciale et la lisibilité de l'offre grâce à des itinéraires en partie ou intégralement dédiés, des dessertes cadencées et des capacités d'accueil augmentées (Gart, 2015). L'arrivée de cette nouvelle offre est associée au réaménagement des abords de la gare de Périgueux. Dans le scénario de desserte retenu en 2013 par l'intercommunalité, le tracé de la ligne BHNS traverse la commune de Périgueux d'est en ouest et dessert la gare de Périgueux à l'ouest des voies ferrées, du côté opposé au bâtiment voyageurs, sur des espaces non encore aménagés. Ce tracé permettait un accès plus facile au centre de Périgueux compte tenu des aménagements routiers existants ${ }^{14}$.

D'autre part, un projet de restructuration de l'offre ferroviaire régionale dans l'agglomération est porté par la Région. Discuté depuis plus de trente ans entre la Région et les collectivités locales, ce projet est formalisé en 2013 dans le contrat d'axe ferroviaire de la Vallée de l'ssle. Dans ce contrat, la Région Aquitaine s'engage à augmenter et cadencer l'offre ferroviaire sur l'axe Mussidan-Niversac, qui dessert la gare de Périgueux et qui traverse la partie nord de l'agglomération ${ }^{15}$. Cet engagement est associé à la mise en œuvre de politiques locales vertueuses en matière d'intermodalité et d'urbanisme dans les communes traversées par l'axe ferroviaire (Maulat, 2015). L'objectif de la Région est ici de développer l'offre en transports collectifs dans cet espace qui concentre un quart de la population et un tiers des emplois du département de la Dordogne ${ }^{16}$. Dans le contrat d'axe, cette recomposition de l'offre est présentée comme un facteur favorable à la hausse du nombre de voyageurs. Elle est aussi associée au redimensionnement des abords de la gare de Périgueux et d'autres gares de l'axe dans le but d'améliorer l'accès au train. Les réaménagements des abords des deux autres haltes existantes (Niversac et Razac) sont notamment compris dans le contrat, ainsi que la construction de deux nouvelles haltes en première couronne de Périgueux (dans la zone industrielle de Boulazac et à Marsac).

A une échelle plus fine, l'aménagement du pôle d'échanges est un outil pour mettre en œuvre la nouvelle politique de report modal de l'intercommunalité. La réduction de la circulation automobile est associée à l'augmentation de la fréquentation des transports collectifs : il s'agit d'encourager les automobilistes à se reporter sur ces deux offres pour réaliser leurs déplacements, notamment vers Périgueux. Ce projet est complémentaire avec d'autres projets en cours pour faciliter le rabattement vers le BHNS et le train, notamment dans les communes limitrophes (parkings relais aux arrêts de bus, nouvelles haltes ferroviaires, etc.). Cette stratégie confère dès lors un nouveau rôle aux transports collectifs dans la planification des déplacements : historiquement peu fréquentés et dédiés à une clientèle captive (Gart, 2006), ils sont désormais considérés par l'intercommunalité comme la première alternative à la voiture individuelle.

\section{Le fer de lance d'une politique multimodale}

En parallèle, l'aménagement d'un pôle d'échanges en gare de Périgueux vise aussi à faciliter les correspondances entre l'ensemble des modes de transports, et notamment entre ces deux réseaux (BHNS et TER) ${ }^{17}$. Avec ce projet, le Grand Périgueux a pour ambition d'augmenter les pratiques de déplacement basées sur l'intermodalité, c'est-à- 
dire sur l'utilisation successive de plusieurs modes au cours d'un déplacement. Ces nouvelles pratiques sont considérées comme un moyen de développer l'usage des solutions alternatives et de réduire les trajets porte à porte réalisés en voiture individuelle. Le site de la gare de Périgueux a été choisi par l'intercommunalité pour aménager un pôle d'échanges car il est un point de convergence entre les modes de transports et le principal accès au réseau ferroviaire de l'agglomération. Le périmètre de projet couvre de larges emprises situées autour du bâtiment voyageurs, des deux côtés des voies ferrées. Il se compose de trois espaces: les espaces de desserte et de stationnement existants déjà aménagés à proximité du bâtiment voyageurs (parvis, parking voiture, parking vélo, voiries, etc.), la passerelle de franchissement des voies ferrées et les espaces situés à l'ouest des voies sur une friche ferroviaire, qui seront à proximité du futur arrêt de la ligne BHNS (figure 7). Le pôle d'échanges vise, d'une part, à transformer les espaces propres à chaque mode au regard des dysfonctionnements existants ${ }^{18}$ et des projets en cours, et d'autre part, à aménager de nouvelles conditions de correspondances, notamment de part et d'autre des voies ferrées.

Figure 7. Périmètre du projet de pôle d'échanges aux abords de la gare de Périgueux.

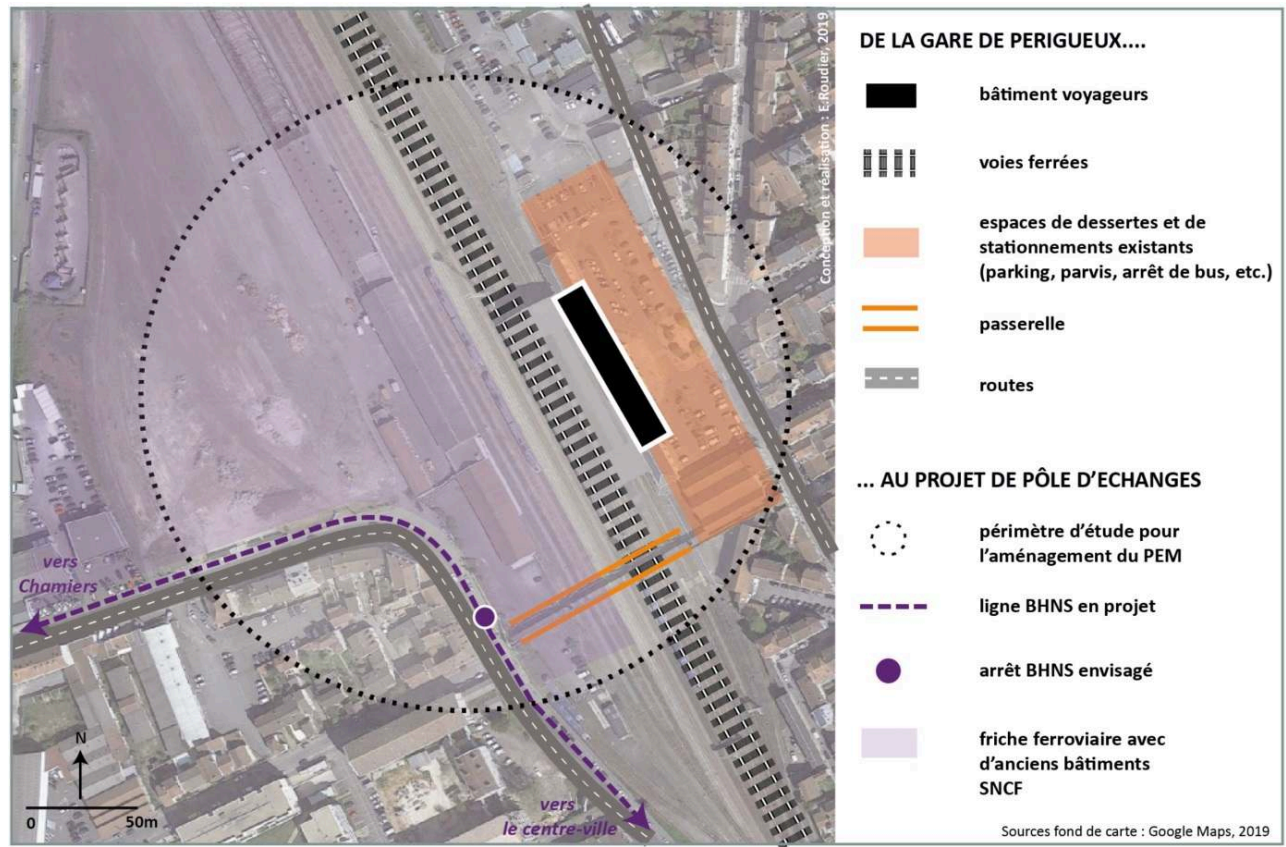

Emilie Roudier, 2020

Le lancement de ce projet montre que l'intercommunalité adopte une nouvelle approche de la mobilité basée sur la complémentarité entre les modes de transport. Audelà du seul projet de pôle d'échanges, le Grand Périgueux associe la mise en œuvre de cette nouvelle approche au lancement de plusieurs projets pour changer simultanément les offres et les aménagements de tous les modes de transport dans l'agglomération. En 2014, des études sont notamment conduites par l'intercommunalité pour élaborer de nouveaux documents de planification (schéma directeur cyclable et piéton, plan de circulation, plan global de déplacements), pour favoriser les solutions alternatives (aménagement de places piétonnes, voies dédiées aux BHNS, pistes 
cyclables) et pour réduire la circulation automobile (contournement routier, réduction du nombre de voies et de parkings, limitation de vitesse).

\section{L'instrument d'une nouvelle coordination urbanisme et transport}

Au-delà des seuls enjeux de mobilité, l'aménagement du pôle d'échanges s'inscrit aussi dans une stratégie de redynamisation de l'ensemble du quartier de la gare de Périgueux. Depuis les années 1980, le quartier connaît une déprise démographique et économique, comme en témoigne l'augmentation des terrains et des établissements en friche ${ }^{19}$. Dans la deuxième partie du $\mathrm{XX}^{\mathrm{e}}$ siècle, le double phénomène de désindustrialisation et de déclin de la fréquentation du transport ferroviaire, observé à l'échelle nationale face à la démocratisation de la voiture, fragilise les activités des quartiers de gare (Delage, 2013 ; Baron, Messulam, 2017). Cette crise du quartier de la gare de Périgueux est aussi liée, à l'échelle locale, à la crise d'attractivité de la commune face aux communes périurbaines.

Face à cette situation, le pôle d'échanges est un outil pour changer cette image dégradée du quartier de la gare, situé à l'ouest des voies ferrées. Son aménagement vise à améliorer les conditions d'accès à la gare mais aussi aux futurs aménagements prévus dans le quartier pour lui redonner de l'attractivité. Le projet de pôle d'échanges s'articule ainsi avec deux autres projets urbains prévus dans le quartier (figure 8). Un projet de centre d'activités tertiaires est porté par le Grand Périgueux, en charge du développement économique, près du futur arrêt BHNS. Un projet de requalification du quartier est conduit par la Ville de Périgueux, en partenariat étroit avec la communauté d'agglomération, pour aménager des logements, équipements et commerces, sur les emprises à proximité du futur centre tertiaire.

Figure 8. Le projet de pôle d'échanges : un outil pour redynamiser le quartier de la gare.

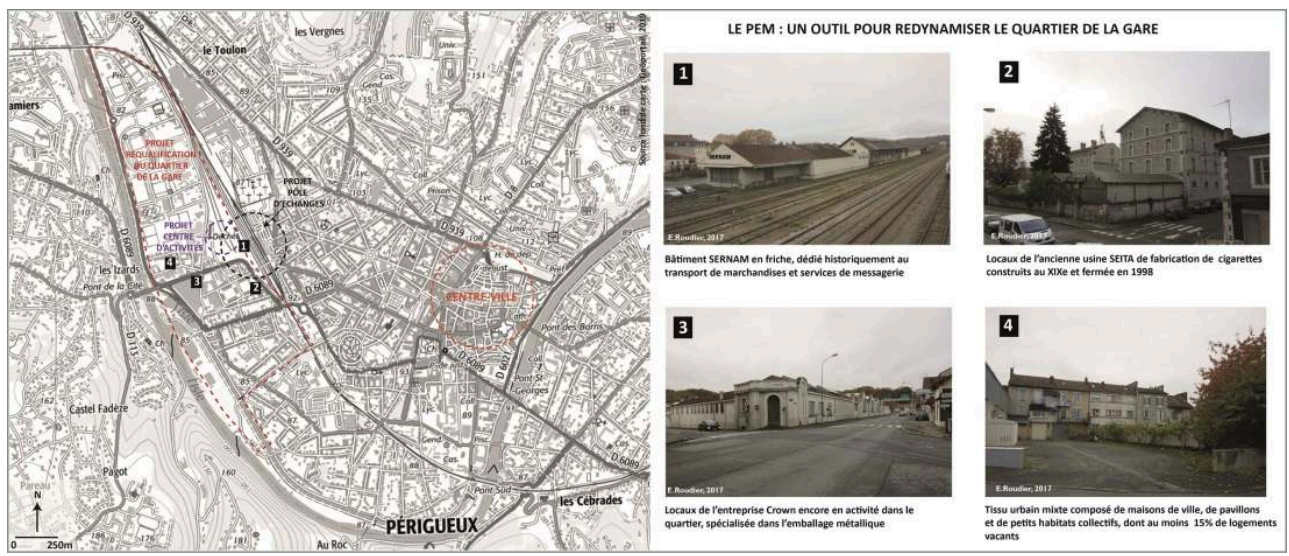

Emilie Roudier, 2020

L'articulation de ces trois projets révèle précisément la nouvelle mise en cohérence entre les projets de transport et les projets d'aménagement urbain à l'échelle de l'agglomération. La coordination entre le développement urbain et l'offre de transports est un nouveau référentiel de la politique locale dans le but de réduire la circulation automobile et de limiter le développement urbain dans des espaces non desservis par des offres en transports collectifs (Gallez et al., 2015). Ce référentiel est également au centre $\mathrm{du}$ contrat d'axe ferroviaire: l'engagement $\mathrm{du}$ renforcement de l'offre 
ferroviaire est conditionné à la mise en œuvre de politiques locales favorables à l'intermodalité et à la densification autour des gares (Maulat, 2015).

L'analyse des conditions de lancement du projet de pôle d'échanges met en évidence un triple changement de référentiels de la politique locale en faveur du report modal, de l'intermodalité et de la coordination urbanisme et transport. Le pôle d'échanges matérialise dans l'espace ces trois référentiels et leurs interdépendances et, plus largement, la mise au premier plan de la mobilité dans les politiques locales à partir de 2014.

\section{Des ambitions à la mise en action : la nouvelle politique locale et ses écueils}

Si le projet de pôle d'échanges est bien au centre de la politique locale de déplacement périgourdine, il n'est pas pour autant une panacée aux problématiques de mobilité locales. La conduite de ce projet révèle aussi les écueils de cette nouvelle politique et les contraintes auxquelles est confrontée l'intercommunalité pour mettre en œuvre ces nouveaux référentiels. Face à la pression de la voiture, le Grand Périgueux disposait déjà de marges de manœuvre restreintes, qui sont d'autant plus affaiblies par des contraintes financières, techniques et politiques.

\section{D'une politique volontariste à une politique en ordre dispersé ?}

Le Grand Périgueux est confronté à des contraintes techniques pour mettre en œuvre sa nouvelle politique locale de déplacement. Dès 2014, la communauté d'agglomération lance pour cela de multiples projets, imbriqués les uns avec les autres, mais elle est confrontée à des difficultés pour les coordonner et les conduire en parallèle. La conduite du projet de pôle d'échanges met précisément en évidence les écueils de cette stratégie.

En étant à l'interface de l'ensemble des modes, le pôle d'échanges est un point d'articulation entre différents projets en cours conduits par l'intercommunalité, comme la ligne BHNS, le plan de circulation ou le schéma directeur cyclable et piéton évoqués précédemment. L'absence de finalisation de ces projets a complexifié, voire freiné, la programmation des aménagements du pôle d'échanges. Lors des études, le tracé de l'offre BHNS et la restructuration du réseau de bus urbain n'étaient pas encore définitifs et il était en conséquent difficile de réaliser des scénarios d'aménagement précis des espaces situés à l'ouest des voies ferrées. De même, à l'échelle du quartier, le manque de stabilisation des programmes du centre d'activités et du quartier de gare a également ralenti le travail sur le dimensionnement des flux attendus. Au cours des études du pôle d'échanges, des équipements à vocation touristiques prévus à proximité de l'arrêt BHNS ont notamment été déplacés dans le centre-ville ${ }^{20}$. Face à ces difficultés, l'intercommunalité a allongé la durée des phases d'études et recentré le périmètre de projet à l'ouest des voies ferrées en privilégiant une transformation progressive des abords de la gare plutôt qu'une transformation globale à court terme, des deux côtés des voies ferrées ${ }^{21}$.

Un plan global de déplacement (PGD) a aussi été réalisé par l'intercommunalité pour répondre à ces contraintes techniques et déterminer la feuille de route de sa politique 
de déplacement ${ }^{22}$. Le PGD est composé d'un plan d'action et matérialise la cohérence et les imbrications entre les différents projets de mobilité et d'aménagement urbain prévus à proximité de la gare. Il a toutefois été finalisé en 2016, soit deux ans après le lancement du projet de pôle d'échanges. Cette situation montre aussi que la mise en œuvre des politiques de déplacement dans les villes moyennes ne peut se résumer uniquement à une succession de projets et qu'elle nécessite une planification fine des actions à engager et une analyse précise des pratiques de déplacement.

\section{De la crise des transports à la crise des finances publiques : les collectivités locales dos au mur?}

En parallèle, l'intercommunalité est également confrontée à des contraintes financières pour conduire sa politique locale de déplacement. Les capacités de financement de l'intercommunalité demeurent limitées face à la contraction de leurs dépenses publiques (Diact, 2009), même si la planification des mobilités est la priorité financière du Grand Périgueux.

37 La conception du projet de pôle d'échanges met en évidence le décalage entre cette vulnérabilité financière et les ambitions de l'intercommunalité. La réalisation de ce type de projet est généralement associée à des budgets de plusieurs millions d'euros, même dans les petites villes (Cerema, 2012). Lors des études, le Grand Périgueux partageait, avec la Ville de Périgueux, l'objectif d'aménager une nouvelle passerelle audessus des voies ferrées pour valoriser, entre autres, l'arrivée du BHNS et les projets urbains prévus dans le quartier. Cette ambition s'est confrontée aux stratégies financières des partenaires. Pour la SNCF et la Région, cet aménagement n'était pas une priorité financière compte tenu de son coût élevé, des contraintes d'exploitation ferroviaire que poserait sa construction et de l'état de la passerelle existante, encore fonctionnelle. Face à cette opposition et à l'incapacité de financer cet aménagement avec la commune, l'intercommunalité a révisé son ambition et s'est alignée sur les stratégies de la Région et de la SNCF.

Ces contraintes financières ne sont ainsi pas un frein à l'action mais elles rendent les collectivités locales plus dépendantes des stratégies de financement des partenaires. Cette situation se trouve par ailleurs dans la mise en œuvre du plan d'actions du plan global de déplacement, associée à un budget total de 47 millions d'euros entre 2017 et $2020^{23}$. Ce budget n'est pas basé uniquement sur des investissements internes de la communauté d'agglomération. Il est surtout conditionné à l'augmentation du versement transport versé par les entreprises du territoire et à la mobilisation de financements externes potentiels (fonds européens, aides régionales et départementales). Cette situation montre que la définition et la mise en œuvre de la politique locale ne peut ainsi être uniquement basée sur des ambitions de l'intercommunalité.

\section{La politique de déplacement locale (trop) interdépendante de la politique régionale?}

Enfin, la mise en œuvre de la politique de déplacement est aussi compromise par des contraintes politiques, notamment de la part de la Région. La politique de report modal du Grand Périgueux est basée en grande partie sur le renforcement de l'offre TER, 
qualifiée de "navette ferroviaire » par les acteurs locaux. Cette stratégie s'inscrit dans la continuité du contrat d'axe ferroviaire signé en 2013 et vise à favoriser l'usage du train dans l'agglomération.

Le projet de pôle d'échanges illustre ainsi les enjeux et les écueils de cette dépendance entre la politique locale et la politique régionale. Lors des études pour la programmation du pôle d'échanges, la question de l'arrivée de la navette ferroviaire était au centre des débats entre le Grand Périgueux et la Région. Alors que l'intercommunalité souhaitait coordonner le pôle d'échanges et le renforcement de l'offre TER, la Région ne pouvait s'engager formellement sur une date de mise en service face à ses propres contraintes financières et politiques, en pleine réforme des périmètres régionaux ${ }^{24}$. Cette situation met en évidence que les deux collectivités ont des représentations différentes du contrat d'axe. Pour l'intercommunalité, le renforcement de l'offre TER était un élément déclencheur du projet de pôle d'échanges, qui devait être coordonné avec l'arrivée de la navette. Pour la Région, il était surtout un " coup politique $»^{25}$ pour acter ce projet vieux de trente ans et non un projet avancé sur les plans techniques et financiers. La navette ferroviaire et le projet de pôles d'échanges étaient même pour la Région des projets distincts, non directement liés ${ }^{26}$.

Face au report de la mise en service de la navette, le Grand Périgueux a révisé et adapté les objectifs du pôle d'échanges. D'une part, le calendrier de réalisation du projet s'est au fur et à mesure désynchronisé de l'arrivée de la navette ferroviaire, désormais prévue pour 2022 (figure 9). D'autre part, le pôle d'échanges est désormais pour l'intercommunalité moins un outil technique et plus un outil politique pour montrer ses efforts politiques et financiers et pour négocier l'avancée du projet de navette ferroviaire portée par la Région.

Figure 9. Le pôle d'échanges en gare de Périgueux : premiers aménagements à l'ouest des voies ferrées.

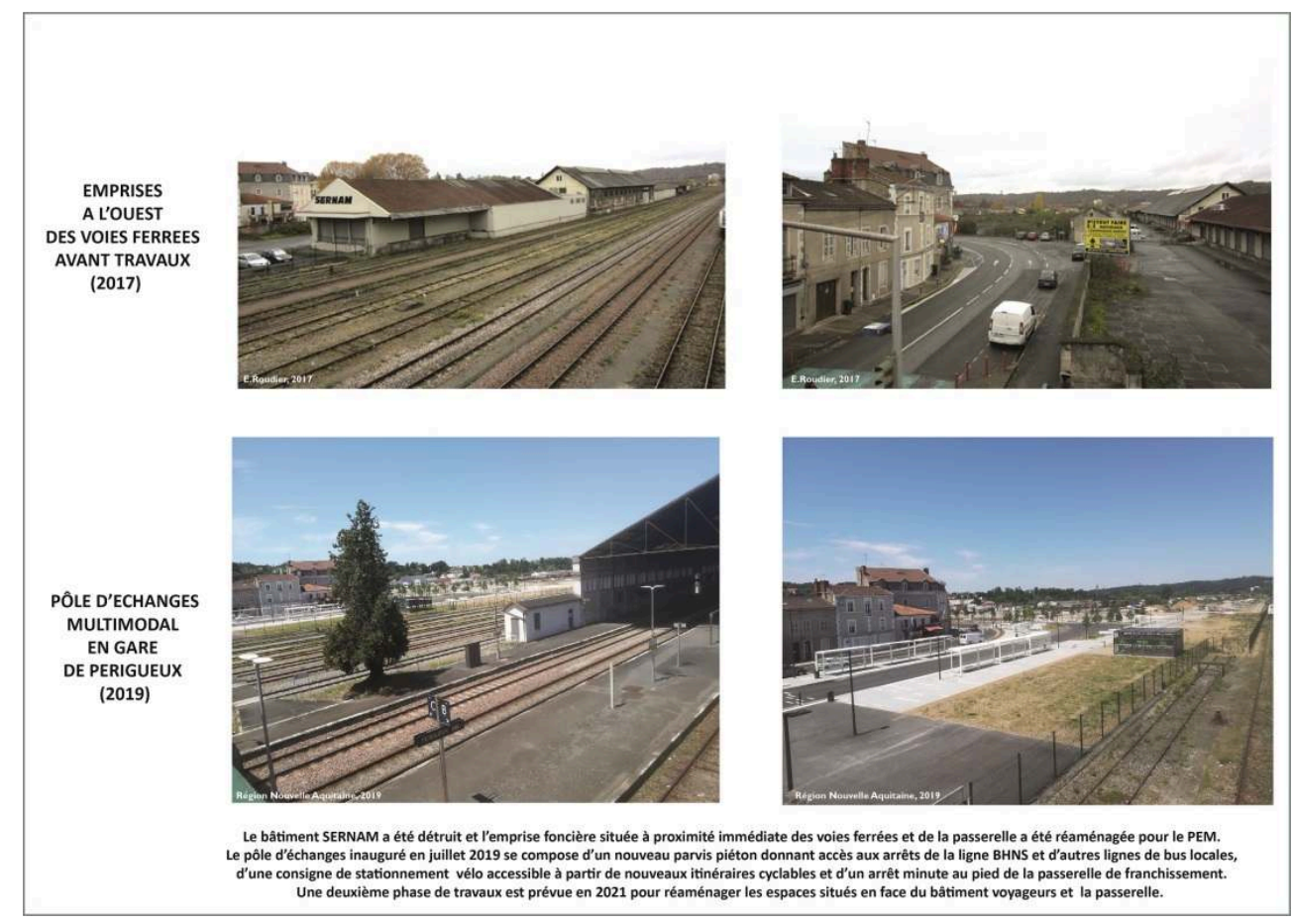

Emilie Roudier, 2020 


\section{Conclusion}

Cet article propose une grille de lecture exploratoire des mutations de la politique locale de déplacement de l'agglomération périgourdine à partir de l'analyse du projet de pôle d'échanges. Cette grille s'appuie sur une étude de cas qui se caractérise par des spécificités locales: un maillage ferroviaire plutôt dense pour une agglomération de cette taille, un projet de bus à haut niveau de services et un positionnement territorial entre deux métropoles régionales. Elle peut toutefois être confrontée à d'autres villes moyennes de taille similaire, qui partagent les mêmes problématiques locales et qui ont lancé un projet de pôle d'échanges.

La conduite du projet de pôle d'échanges cristallise les principales évolutions de la politique locale de déplacement périgourdine depuis dix ans. Elle révèle les basculements des représentations de la voiture et des transports collectifs de la part des acteurs locaux ainsi que les changements de mode d'action de l'intercommunalité, qui s'engage désormais dans une planification locale des mobilités. Elle illustre surtout les changements de référentiels politiques en faveur du report modal, de l'intermodalité et de la coordination urbanisme et transport. Ces référentiels ne sont toutefois pas propres à l'agglomération périgourdine ni aux villes moyennes. Ils sont largement encouragés par les politiques publiques depuis vingt ans mais force est de constater que ces agglomérations les prennent en compte tardivement, plus motivées par l'augmentation des nuisances locales que par les multiples injonctions nationales à la mobilité durable.

Le projet de pôle d'échanges constitue une réponse aux problématiques locales de Périgueux, et, plus largement, de nombreuses villes moyennes qui choisissent de lancer ce type de projet dans leur territoire. Cette réponse est cependant sous contrainte et sans doute incomplète, à l'image de la politique locale de déplacement. La mise en œuvre de ce type de projet est associée à des contraintes financières, techniques et politiques, qui sont emblématiques des villes moyennes. Ces contraintes tendent à limiter les ambitions politiques de l'intercommunalité et à recomposer leurs objectifs de projets, notamment face aux stratégies différenciées des régions et des acteurs ferroviaires.

Depuis dix ans, la politique de déplacement périgourdine est ainsi prise en étau entre la prise de conscience des problématiques locales, le volontarisme politique de l'intercommunalité et les freins à la réalisation de leurs ambitions. Cet article montre toutefois que face à ces contraintes, les intercommunalités font preuve de capacités d'adaptation pour assurer un passage à l'action et pour au moins engager une évolution des aménagements et des pratiques sur le territoire.

Cette analyse invite à continuer à étudier ces évolutions politiques, notamment dans le cadre des projets inscrits dans le plan «Action cœur de ville». Ces nouveaux projets sont de nouvelles occasions de réfléchir sur les réponses à apporter aux maux de la mobilité dans les villes moyennes et ils peuvent constituer des laboratoires pour révéler de nouvelles ruptures ou transitions dans les politiques locales de déplacement des villes moyennes. 


\section{BIBLIOGRAPHIE}

BARON N., MESSULAM P. (2017), Réseaux ferrés et territoires : La géographie humaine du chemin de fer. Un retour aux sources, $368 \mathrm{p}$.

BEHAR D. (2011), « Bricolage stratégique et obligation d'innovation », Urbanisme, 378, pp. 50-51.

BEHAR D. (2018), «Crise des villes moyennes, une question géopolitique », Acteurs de l'économie, $74 \mathrm{p}$.

CEREMA (2012), « Mettre les gares TER au cœur des stratégies territoriales : pourquoi ? comment? ", Stratégies foncières aux abords des gares TER, pp. 1-8.

CEREMA (2019), Mobilité dans les villes moyennes - 3 échelles territoriales d'analyse, Editions du Cerema, $112 \mathrm{p}$.

CHADEFAUD M. (1963), « Les industries de Périgueux », in Revue géographique des Pyrénées et du Sud-Ouest, 34, pp. 405-409.

CHARMES E. (2017), « La revanche des villages », La vie des idées.

DELAGE A. (2013), La gare, assurance métropolitaine de la ville post-industrielle. Le retournement de valeur dans les projets urbains de quartier de gares à Saint-Etienne Châteaucreux (France) et LiègeGuillemins (Belgique), Thèse en géographie, aménagement de l'espace et urbanisme, Université Lyon 2.

DELPIROU A. (2019), « Action cœur de ville ou les plafonds de verre de l'action publique territoriale », Horizons publics, été 2019, pp. 44-51.

DEMAZIÈRE C. (2014), « Pourquoi et comment analyser les villes moyennes ? Un potentiel pour la recherche urbaine », Métropolitiques, 1, dossier « Villes moyennes ».

DE ROO P. (2005), Livre Blanc. Villes moyennes, villes d'intermédiation. Pour une diversité des modèles de développement urbain, Paris, DATAR, décembre, $160 \mathrm{p}$.

DELEGATION INTERMINISTERIELLE A L'AMENAGEMENT ET A LA COMPETITIVITE DES

TERRITOIRES (DIACT), (2009), Les villes moyennes françaises : enjeux et perspectives, La documentation française.

DI MEO G. (1971), « Périgueux : la population, l'urbanisation récente et ses problèmes », Revue géographique des Pyrénées et du Sud-Ouest, pp. 181-196.

DUPUY G. (1999), La dépendance automobile. Symptômes, analyses, diagnostic, traitements, Paris, Edition Anthropos, 160 p.

ELOI J.-S. (2005), Le monde cheminot à Périgueux : une communauté perdue, Editions Fanlac.

GALLEZ C. (2007), « Intercommunalité, transports urbains et pouvoir d'agglomération. Cinq trajectoires urbaines », Flux, 68, 2, pp. 43-61.

GALLEZ C et al. (2015) «Le rôle des outils de coordination urbanisme-transports collectifs dans la fabrique politique urbaine », Flux, 101-102, pp 5-15.

GART, FMVM, FNAUT (2006), Actes du colloque « Les déplacements dans les villes moyennes : comment améliorer l'usage des transports collectifs? ", $40 \mathrm{p}$.

GART (2015), Mobilité : état des lieux et perspectives, Paris, Editions du GART, 86 p. 
GENTY M. (1981), « Le désenclavement routier et ferroviaire des villes du Périgord et du Bas-Pays limousin au XIX ${ }^{\mathrm{e}}$ siècle », in Annales du Midi : revue archéologique, historique et philologique de la France méridionale, 93, 153, pp. 279- 291.

HAYS D. (1993), « Commerces de détail et grandes surfaces à Périgueux et sa banlieue ", in Bulletin de l'Association de géographes français, 70e année, Commerce et aménagement. pp. 199-208.

HERAN F. (2017), «Vers des politiques de déplacements urbains plus cohérentes », Norois, 4, 245, pp. 89-100.

LE GRAND PERIGUEUX (2016), Plan global de déplacements Périmouv, 42 p.

LE GRAND PERIGUEUX (2014), Cahier des clauses techniques et particulières étude de programmation et de faisabilité du pôle d'échanges multimodal de la gare de Périgueux, 13 p.

LEJOUX P. (2017), « Les petites villes face à la montée des contraintes énergétiques :

l'augmentation du coût de la mobilité, un facteur de vulnérabilité économique ? ", Territoire en mouvement. Revue de géographie et aménagement, 33.

MAULAT J. (2015), « Contractualiser pour coordonner urbanisme et transport ? Regards croisés sur quatre expériences de contrats d'axes ferroviaires », Flux, 101-102, 3, pp. 82-98.

MAULAT J., PASSALAQUA A. (2014), « Les collectivités locales prises dans la nouvelle "bataille du rail" », Métropolitiques.

MENERAULT P. (2006), Les pôles d'échanges en France : état des connaissances, enjeux et outils d'analyse, Editions du Certu, $180 \mathrm{p}$.

PINSON G. (2005), « Le projet urbain comme instrument d'action publique », in Gouverner par les instruments, Presses de Sciences-Po, pp. 199-233.

REGION AQUITAINE (2013), Contrat d'axe de la Vallée de l'Isle, 26 p.

RICHER C. (2008), «L'émergence de la notion de "pôle d'échanges" : entre interconnexion des réseaux et structuration des territoires ", Les Cahiers Scientifiques du Transport, 54, pp. 101-123.

ROUDIER E. (2019), Quand la ville moyenne entre en gare: des projets entre mimétisme métropolitain et recompositions territoriales, Thèse en urbanisme et aménagement de l'espace, Université Paris-Est Créteil.

SANTAMARIA F. (2012), « Les villes moyennes françaises et leur rôle en matière d'aménagement du territoire : vers de nouvelles perspectives? ", Norois, 2, 223, Presses Universitaires de Rennes, pp. 13-30.

TESSON F. (2002), « Autoroute et recompositions territoriales : la mise en lumière de quelques paradoxes à travers l'exemple de la Dordogne », Géocarrefour, 77, 1, pp. 55-64

VILLE DE PERIGUEUX (2014), Plan local d'urbanisme.

\section{NOTES}

1. Élaboré en concertation avec l'association Villes de France, les élus locaux et les trois partenaires financiers nationaux du programme (la Banque des territoires, Action logement et l'Agence nationale de l'habitat), le programme vise à faciliter et à soutenir le travail des collectivités locales, à inciter les acteurs du logement, du commerce et de l'urbanisme à réinvestir les centres-villes, à favoriser le maintien ou l'implantation d'activités en cœur de ville, 
afin d'améliorer les conditions de vie dans les villes moyennes. Source : https://www.cohesionterritoires.gouv.fr/programme-action-coeur-de-ville.

2. En 2005 , 98 plans de déplacements urbains volontaires, réalisés par des agglomérations de moins de 100000 habitants, ont notamment été recensés par le GART (Gart, 2006).

3. Loi 2000-1208 du 13 décembre 2000 relative à la solidarité et au renouvellement urbains.

4. Loi 2010-788 du 12 juillet 2010 portant engagement national pour l'environnement.

5. Cette enquête a été réalisée dans le cadre d'une thèse en convention Cifre (Convention Industrielle de Formation à la Recherche) avec le bureau d'études Kisio et le laboratoire Lab'Urba (Université Paris-Est) de 2015 à 2019.

6. Devant Bergerac (27 269 habitants) et Sarlat-la-Canéda (8 946 habitants) selon les données de l'INSEE (2016).

7. Ville de Périgueux, (2014), Plan local d'urbanisme.

8. LE GRAND PERIGUEUX (2016), Plan global de déplacements Périmouv, $42 \mathrm{p}$.

9. Entretien avec un élu de la Ville de Périgueux, janvier 2017.

10. LE GRAND PERIGUEUX (2016), Plan global de déplacements Périmouv, 42 p.

11. La gare de Périgueux est une gare réceptrice en heures de pointe du matin: il y a plus de personnes qui sortent de la gare de Périgueux le matin en heures de pointe pour se rendre à leur lieu de travail ou leur lieu d'études que de personnes qui vont prendre le train à Périgueux pour se déplacer ensuite en dehors de la commune.

12. Il est rare que les agglomérations de cette taille aient autant de gares sur leurs territoires. A titre de comparaison, l'agglomération de St. Omer n'en possède que deux, de même pour l'agglomération de Saintes (Roudier, 2019).

13. La Communauté d'Agglomération du Grand Périgueux créée au 1er janvier 2014 est à l'origine la fusion de deux intercommunalités: la communauté d'agglomération périgourdine et la communauté de communes Isle Manoire en Périgord. Elle se compose à l'origine de 34 communes et s'élargit à nouveau au 1er Janvier 2017 avec un périmètre de 43 communes.

14. Entretien avec un technicien du Grand Périgueux, septembre 2017.

15. REGION AQUITAINE (2013), Contrat d'axe de la Vallée de l'Isle, 26 p.

16. Entretien avec un élu de la Région Aquitaine, avril 2017.

17. LE GRAND PERIGUEUX (2014), Cahier des clauses techniques et particulières étude de programmation et de faisabilité du pôle d'échanges multimodal de la gare de Périgueux, $13 \mathrm{p}$.

18. La saturation des parkings, la faible multimodalité du parvis entièrement dédié à l'automobile et l'insuffisance de l'information voyageurs sont autant de freins à l'intermodalité préalablement identifié par l'intercommunalité.

19. Ville de Périgueux (2014), Plan local d'urbanisme.

20. A l'origine, la construction d'un équipement à vocation touristique et économique, la «Manufacture Gourmande » était prévu dans le quartier de gare, à proximité de l'arrêt du nouveau bus à haut niveau de service. La programmation de l'équipement a été déplacée par la Ville dans le centre-ville, à proximité de la cathédrale.

21. Les premiers aménagements à l'ouest des voies ferrés ont été inaugurés en 2019.

22. Sur le modèle du PDU, des démarches volontaires peuvent être réalisées pour les collectivités de taille inférieure. Les PDU-volontaires et plan global de déplacement (PGD) reprennent les mêmes orientations générales que les PDU. Ce plan constitue un cadre de réflexion et de proposition de principes pour la réorganisation de l'ensemble des déplacements à l'échelle d'un territoire sur une durée de 10 ans.

23. Article "Grand Périgueux : un plan de déplacement à 47 millions d'euros d'ici 2020 », SudOuest, $28 / 10 / 16$

24. Les études du pôle d'échanges se sont déroulées entre décembre 2014 et septembre 2015, soit quelques mois avant la réforme des périmètres régionaux au 1e janvier 2016 (loi du 16 janvier 2015 relative à la délimitation des régions). 
25. Entretien avec un élu de la Région Aquitaine, avril 2017.

26. Entretien avec un technicien de la Région Aquitaine, octobre 2016.

\section{RÉSUMÉS}

Cet article s'intéresse aux mutations des politiques locales de déplacements dans les villes moyennes depuis les années 2000. Il étudie plus précisément le cas de la politique menée à Périgueux, ville moyenne de la Dordogne, par la Communauté d'Agglomération du Grand Périgueux. Il propose d'étudier ces mutations par le prisme du projet de pôle d'échanges en gare de Périgueux. A partir de l'analyse des conditions de lancement et de conception de ce projet, l'article rend compte des changements de paradigmes de la politique locale périgourdine et des contraintes politiques, techniques et financières auxquelles se confrontent l'intercommunalité pour les mettre en œuvre. Cette étude de cas est l'occasion de poser la question de la planification des mobilités dans les villes moyennes en mettant en perspective les principales problématiques locales, les évolutions récentes des référentiels et des modes d'action et les limites des capacités d'action des collectivités locales.

This paper analyses the mutations of mobility policies in French mid-sized cities since the 2000s. It studies the case of Périgueux, a mid-sized city in Dordogne, and its inter-municipal transport policies. It proposes to analyze these mutations through the prism of the Périgueux interchange train station project, a major project of the inter-municipality. Through a study of the launch and design of this project, the article shows paradigmatic shifts in local policies and political, technical and financial implementation constraints. This case study is an opportunity to question mobility and transport in mid-sized cities. It puts in perspective local issues, evolutions of frameworks and practices and the limits of local authorities capacity for action.

\section{INDEX}

Keywords : mid-sized cities, mobility, interchange station, local, crisis, Périgueux

Mots-clés : villes moyennes, mobilité, pôle d'échanges, politique locale, crise, Périgueux

\section{AUTEUR}

\section{EMILIE ROUDIER}

Lab'urba - Université Paris-Est, eroudier.rch@gmail.com 